\title{
Polymorphisme des régions capside et 3'NTR de 3 isolats tunisiens du virus $Y$ de la pomme de terre (PVY)
}

\author{
H Fakhfakh ${ }^{1 *}$, M Makni ${ }^{1}$, C Robaglia 2, A Elgaaied ${ }^{1}$, M Marrakchi ${ }^{1}$ \\ 1 Laboratoire de génétique et biologie moléculaire, faculté des sciences de Tunis, campus universitaire, \\ 1060 Le Belvedère, Tunis, Tunisie; \\ 2 INRA, laboratoire de biologie cellulaire, route de Saint-Cyr, F-78026 Versailles, France
}

(Reçu le 11 mai 1995 ; accepté le 10 novembre 1995)

\begin{abstract}
Résumé - En Tunisie, le virus $Y$ de la pomme de terre (PVY) occasionne des pertes importantes sur la production des cultures maraîchères, en particulier le piment. Le séquençage de la région $3^{\prime}$ non codante ( $3^{\prime} N T R$ ) et de la région codant pour la protéine capside (CP) de 2 isolats de PVY d'origine piment et d'un isolat d'origine pomme de terre semble indiquer une corrélation entre les manifestations symptomatologiques de ces isolats et leurs propriétés moléculaires. La structure secondaire potentielle de l'ARN viral au niveau de la région 3'NTR montre des différences entre les 3 isolats. L'analyse de la séquence de la région codant pour la protéine capside suggère qu'un phénomène de recombinaison serait à l'origine de l'isolat d'origine piment PVY-P21.
\end{abstract}

PVY / 3'NTR / structure secondaire / capside

Summary - Sequence polymorphism in the 3'-NTR and the CP protein of 3 Tunisian isolates of the potato virus $Y$. In Tunisia, potato virus $Y(P V Y)$ causes yield losses, particularly on pepper cultures. Sequence analysis from the 3'-non-translated region ( $3^{\prime}-\mathrm{NTR}$ ) and from the coat protein coding region (CP) of 2 PVY isolates from pepper and 1 from potato are in agreement with the biological classification. Potential RNA secondary structure analysis of the 3 '-NTR region revealed additional differences between isolates. Phylogenetic analysis of these 3 sequences suggests that 1 isolate (PVY-P21) might have emerged through a recombination process.

PVY / 3'-NTR / secondary structure / CP

\section{INTRODUCTION}

En Tunisie, l'agriculture occupe une place de choix dans l'économie du pays. La production des cultures maraîchères a été marquée, ces dernières années, par une baisse considérable due aux maladies d'origine cryptogamique et virale. Le principal agent viral, le virus $Y$ de la pomme de terre (PVY), est le membre type du groupe des potyvirus, le plus important des virus

* Correspondance et tirés à part 
végétaux avec plus de 180 membres (Brunt, 1992). Le génome de ce groupe est constitué d'un ARN simple brin positif d'environ $10 \mathrm{~Kb}$, polyadénylé en $3^{\prime}$ (Hari, 1981) est lié en $5^{\prime}$ de manière covalente à une protéine $(\mathrm{VPg})$ (Hollings et Brunt, 1981).

Les systèmes de traduction in vitro et la détermination des séquences nucléotidiques ont montré l'existence d'une seule phase de lecture ouverte (ORF) conduisant à la synthèse d'un précurseur polyprotéique d'environ 3000 acides aminés. Ce précurseur est clivé par cascade autocatalytique pour générer 7 à 8 protéines virales (Carrington et Dougherty, 1987 ; Carrington et al, 1989; Verchot et al, 1991; Riechmann et al, 1992).

L'identification et la classification des potyvirus reposent essentiellement sur la sérologie et sur la symptomatologie. La gamme d'hôtes du PVY est constituée principalement de solanacées. Chez la pomme de terre, 3 groupes de souches ont pu être définis d'après la symptomatologie sur le tabac, la pomme de terre et sur d'autres plantes hôtes (De Bokx et Huttinga, 1981 ; Le Romancer et al, 1994). Par ailleurs, Gébré Sélassié et al (1985) ont défini plusieurs pathotypes chez les populations virales inféodées au piment. Toutefois, la richesse du groupe ainsi que sa complexité ont engendré de nombreux problèmes de classification pour l'attribution des groupes et des pathotypes à des isolats donnés (Francki, 1983; Francki et al, 1985). Ces problèmes sont dus essentiellement à l'absence de corrélation entre les propriétés biologiques et les relations sérologiques (Francki, 1983). Actuellement, l'analyse des relations phylogénétiques déduites à partir de l'étude des séquences nucléotidiques et d'acides aminés peut conduire à une classification moins controversée.

La comparaison des séquences de la protéine capside de 17 souches de 8 potyvirus différents a montré un pourcentage d'homologie entre différents potyvirus compris entre 38 et $71 \%$; en revanche entre souches d'un même virus I'homologie est de l'ordre de 90 à $99 \%$ (Shukla et Ward, 1988).

L'analyse biologique de 21 isolats tunisiens de PVY collectés sur les cultures de piment (Fakhfakh et al, 1994) a permis de les répartir en 3 groupes :

- un groupe A dont le représentant (un seul isolat) s'attaque à toutes les variétés de piment sensibles et tolérantes mais pas à la variété résistante CM334.
- Un deuxième groupe B (14 isolats) s'attaquant uniquement aux variétés sensibles et induisant des mosaïques et des liserés de nervures.

- Un troisième groupe C (6 isolats) s'attaquant également uniquement aux variétés de piment sensible et montrant, en plus des symptômes observés chez les isolats du groupe $B$, des nécroses.

Dans la nomenclature proposée par Gébré Selassié et al (1985), le groupe A correspond au pathotype $P V Y-1$, les groupes $B$ et $C$ correspondent au pathotype PVY-0.

La séquence des régions $5^{\prime}$ non codante ( $5^{\prime}$ NTR) et de la protéine $P 1$ de 3 isolats tunisiens (PVY-P21, PVY-P2, PVY-Tu) a été déterminée et comparée 2 à 2 avec celle de plusieurs autres isolats de PVY (Marie-Jeanne Tordo et al, 1995). Le pourcentage d'identité nucléotidique de la région $5^{\prime}$ NTR et le pourcentage de similarité en acides aminés de la protéine $\mathrm{P} 1$ ont permis de distinguer 3 groupes phylogénétiques. Les isolats tunisiens constituent, avec d'autres isolats d'origine méditerranéenne collectés sur piment et sur pomme de terre, un seul groupe.

Le présent travail consiste à analyser, chez 3 isolats tunisiens, les séquences de la région codant pour la protéine de la capside et celle de la région $3^{\prime}$ NTR. Cette analyse est entreprise en raison de l'importance de la protéine capsidiaire qui est le déterminant majeur des relations sérologiques. Cette protéine, multifonctionnelle, intervient dans le maintien de la structure de la particule virale et son encapsidation (Shukla et Ward, 1989), dans la vection par les aphides grâce à la présence du motif DAG (Asp-Ala-Gly) situé dans le domaine $\mathrm{N}$ terminal (Atreya et al, 1991) ainsi que dans les mouvements de cellule en cellule (Dolja et al, 1995).

La région $3^{\prime}$ non codante des potyvirus, riche en $A$ et $U$ est une région assez variable en taille et en séquence (Lain et al, 1988 ; Frenkel et al, 1989 ; Turpen, 1989). Elle se termine par la queue poly $(A)$ codée par le génome viral (Dolja et Carrington, 1992). Cette région 3'NTR est impliquée dans l'initiation de la synthèse du brin négatif d'ARN (Bryan et al, 1992 ; Dolja et Carrington, 1992), ainsi que dans la sévérité de l'expression des symptômes (Rodriguez-Cerezo et al, 1991).

Par ailleurs, au niveau de cette région 3'NTR, une différence de séquences assez significative a permis de discriminer entre les divers potyvirus, d'une part, et entre les souches d'un même virus, d'autre part (Frenkel et al, 1989). Les résultats obtenus pour les isolats tunisiens sont discu- 
tés en relation avec certaines différences symptomatologiques.

\section{MATÉRIEL ET MÉTHODES}

\section{Matériel biologique}

\section{Hôtes de multiplication du PVY}

Les isolats tunisiens de PVY collectés sur la variété tunisienne de piment sensible «Beldi» ont été multipliés, après inoculation mécanique en présence d'un abrasif (carborundum), sur les variétés de tabac suivantes : Nicotiana tabacum var xanthi, $N$ glutinosa et $N$ occidentalis.

\section{Isolats viraux utilisés}

Les 3 isolats tunisiens de PVY analysés sont :

- PVY-P2 du groupe B qui induit des mosaïques et des liserés de nervures sur les variétés de piment sensible Beldi et Anaheim ;

- PVY-P21 du groupe C qui provoque, en plus des symptômes sus-cités, des nécroses ;

- PVY-Tu, un isolat prélevé sur pomme de terre mais qui ne provoque aucun symptôme sur les variétés de piment sensible Beldi et Anaheim (Fakhfakh et al, 1994).

Chacun de ces isolats a été cloné biologiquement suite à au moins 2 passages successifs sur Chenopodium amaranticolor.

\section{Méthodes}

\section{Purification du PVY}

Le virus a été purifié selon le protocole de Dougherty et Hiebert (1980) avec quelques modifications. En effet, après précipitation au PEG 6000, le culot renfermant les particules virales est remis en suspension dans le tampon Hepes $20 \mathrm{mM}$ pH 7,5 et laissé sous agitation lente à $4^{\circ} \mathrm{C}$ durant une nuit. Après clarification, les virions sont reprécipités par centrifugation à $110000 \mathrm{~g}$ pendant $2 \mathrm{~h}$. Le culot viral, repris dans le tampon Hepes $20 \mathrm{mM}$, EDTA $2 \mathrm{mM}$ est soumis à une centrifugation à $35000 \mathrm{rpm}$ dans le rotor SW40 sur gradient de saccharose $10-40 \%$ pendant $2 \mathrm{~h}$ à $4^{\circ} \mathrm{C}$. Après fractionnement (ISCO), la bande virale est diluée dans le tampon Hepes/EDTA. Les virions sont centrifugés et le culot repris dans de l'eau stérile.

\section{Extraction de I'ARN viral}

L'ARN viral est extrait en ajustant la solution virale à $1 \mathrm{mg} / \mathrm{ml}$ de protéinase $\mathrm{K}$ et $1 \%$ de SDS. Après extrac- tion au phénol/chloroforme et précipitation à l'éthanol, I'ARN viral est remis en suspension dans de l'eau stérile.

\section{Synthèse d'ADNc des régions capside et $3^{\prime}$ NTR}

Le premier brin d'ADNc capside a été synthétisé grâce à la transcriptase réverse du virus de la myéloblastose aviaire (AMV-TR, Boehringer) en initiant sur I'ARN viral avec un oligonucléotide anti-sens (A), complémentaire des nucléotides 9400-9418 de la séquence de la souche PVYn-Fr (Robaglia et al. 1989). La synthèse du second brin d'ADNc se fait par amplification enzymatique in vitro (PCR) en utilisant la Taq DNA polymérase (Promega), l'oligo anti-sens (A), ainsi que l'oligo sens $(B)$ complémentaire aux nucléotides 8504-8522 de la séquence de la souche PVYn. Fr.

- Oligo antisens (A) : 5' GCATATGTAAATACTTA 3'.

- Oligo sens (B) : 5' GCCTTCACTGAAATGAT 3'.

La région $3^{\prime} N T R$ a été amplifiée grâce à l'oligo sens (B) décrit précédemment et l'oligo (C) comprenant 6 nucléotides complémentaires aux 6 derniers nucléotides du PVY suivis de 15 thymidines:

- oligo (C) : 5' TTTTTTTTTTTTTTTGTCTCC 3'.

La réaction de polymérisation en chaîne (PCR) a été réalisée dans un thermocycler Perkin Elmer selon les conditions suivantes : 2 cycles $\left(94^{\circ} \mathrm{C} 1 \mathrm{mn}, 37^{\circ} \mathrm{C}\right.$ $\left.1 \mathrm{mn}, 72^{\circ} \mathrm{C} 2 \mathrm{mn}\right), 28$ cycles $\left(94^{\circ} \mathrm{C} 1 \mathrm{mn}, 45^{\circ} \mathrm{C} 1 \mathrm{mn}\right.$, $\left.72^{\circ} \mathrm{C} 2 \mathrm{mn}\right)$ et $1 \mathrm{cycle}\left(94^{\circ} \mathrm{C} 1 \mathrm{mn}, 45^{\circ} \mathrm{C} 1 \mathrm{mn}, 72^{\circ} \mathrm{C}\right.$ $10 \mathrm{mn})$.

L'ADNc correspondant à la région capside a été cloné dans le vecteur pBluescript (Stratagene) selon la méthode de Marchuk et al (1990).

\section{Séquençage}

Pour séquencer la totalité de I'ADNc capside, des sous-clones ont été obtenus par la méthode des délétions séquentielles (Steggles, 1989). Le séquençage de ces sous-clones dans un séquenceur automatique (Applied Biosystem) a été réalisé selon la méthode enzymatique (Sanger et al, 1977) en utilisant le kit “Dye Primer». L'ADNc correspondant à la région 3'NTR a été séquencé directement à partir des produits d'amplification PCR en utilisant le kit «Dye Terminator» (Applied biosystem).

La séquence des isolats tunisiens a été comparée avec celle de 6 autres isolats de PVY au niveau de la région $3^{\prime}$ NTR (tableau I) et 7 au niveau de la région capside (tableau II). Les comparaisons et les alignements de séquences en nucléotides et en acides aminés, les cartes de restriction ainsi que les structures secondaires ont été établis grâce aux programmes Seqed, Map, Translate, Gap, Pileup et Fold disponibles dans le serveur GCG (Genetic Computer Group) de I'INRA de Versailles (Devereux et al, 1984). 
Tableau I. Origine géographique et classification des isolats de PVY utilisés pour la comparaison avec les isolats tunisiens au niveau de la région 3 NTR (Pdt: Pomme de terre ; NTN : isolat du type N nécrogène sur tubercules de pomme de terre).

Isolats

PVYNTN-Lib

PVYNTN-M

PVYn-Fr

PVYO-CRNA

PVYNTN-H

PVYO-A
Origine

Liban
Hongrie
France
Japon
Hongrie
Argentine

Hôte

Pdt NTN

Pdt NTN

Pdt $\quad \mathrm{N}$

Pdt NTN

Pdt $\mathrm{O}$
Référence

Le Romancer et al, 1994

Le Romancer et al, 1994

Robaglia et al, 1989

Hidaka et al, 1992

Thole et al, 1993

Bravo-Almonacid et Mentaberry, 1989

Tableau II. Origine géographique et classification des isolats de PVY utilisés pour la comparaison avec les isolats tunisiens au niveau de la région codant pour la protéine capside (Pdt : Pomme de terre ; NTN : isolat du type $N$ nécrogène sur tubercules de pomme de terre).

$\begin{array}{llccl}\text { Isolats } & \text { Origine } & & \text { Hôte } & \text { Référence } \\ & & & & \\ \text { PVYNTN-H } & \text { Hongrie } & \text { Pdt } & \mathrm{NTN} & \text { Thole et al, 1993 } \\ \text { PVYn-Fr } & \text { France } & \text { Pdt } & \mathrm{N} & \text { Robaglia et al, 1989 } \\ \text { PVY-T } & \text { Japon } & \text { Pdt } & \mathrm{N} & \text { Oshima et al, 1991 } \\ \text { PVY-capf } & \text { États-Unis } & \text { Tabac } & \mathrm{O} & \text { Sudarsono et al, 1993 } \\ \text { PVY-capb } & \text { États-Unis } & \text { Tabac } & \text { Potato US } & \text { Sudarsono et al, 1993 } \\ \text { PVY-GO16 } & \text { Allemagne } & \text { Pdt } & \text { O } & \text { Wefels et al, 1989 }\end{array}$

\section{RÉSULTATS ET DISCUSSION}

\section{Comparaison des séquences de la région 3'NTR}

L'alignement de séquences des régions 3'NTR (fig 1) permet de mettre en évidence certaines différences entre les isolats tunisiens. En effet, l'isolat PVY-P2 se distingue des 2 autres par les substitutions en position 83, 110,181, 215 et 247. L'isolat PVY-P21 se caractérise par une insertion en position 13 et l'isolat PVY-Tu présente par rapport aux autres une délétion en position 14.

Afin de définir des critères moléculaires permettant d'évaluer le polymorphisme entre les isolats tunisiens, les séquences de la région $3^{\prime}$ NTR ont été comparées à des séquences d'isolats de PVY disponibles dans les banques de données (tableau I).

Le pourcentage d'identité en nucléotides a permis de distinguer 2 groupes au niveau du dendrogramme produit par le programme Pileup (fig 2). Le groupe (I) est constitué par les 3 isolats tunisiens, qui présentent entre eux une homologie de l'ordre $99 \%$; le groupe (II) est constitué d'isolats du type $\mathrm{O}$ et du type $\mathrm{N}$ dont I'homologie intragroupe varie entre 93 et $99 \%$. Cette analyse montre que les 3 isolats tunisiens sont regroupés, suggérant, comme pour les régions $5^{\prime} N T R$ et $P 1$, qu'ils auraient une origine commune (Marie-Jeanne Tordo et al, 1995). Dans cette comparaison, la souche PVYn-Fr présente le plus faible niveau d'homologie (86-89\%).

L'étude, par le programme Fold (GCG), de la structure secondaire potentielle de l'ARN des isolats tunisiens au niveau de cette région $3^{\prime}$-NTR suggère la présence respectivement de 5,4 et 3 structures "tige-boucle" pour les isolats PVYP21 (fig 3), PVY-Tu et PVY-P2. La séquence de la boucle I (AUAUGCA), située en position 40-42 à partir du codon stop, caractéristique du groupe O (Van der Vlugt et al, 1993), est strictement la même pour les 3 isolats. La séquence des boucles II (UUUCG) et III (UUUUG) situées respectivement en position 134 et 241-243 est identique chez les isolats PVY-P21 et PVY-Tu. L'isolat PVY-P2 présente des structures tigeboucle II et III différentes de celles des autres isolats conduisant à une structure secondaire 
Fig 1. Alignement multiple des séquences en nucléotides de la région $3^{\prime} N T R$ des isolats tunisiens. Les changements de nucléotides sont indiqués en gras et par un astérisque. L'isolat PVY-P2 se caractérise par 5 substitutions par rapport aux 2 autres isolats tunisiens. L'isolat PVY-P21 se caractérise par une insertion en position 13. L'isolat PVY-Tu présente une délétion en position 14.
PVY-Tu TGATTGTGAT GC..CTCTCC GGACGATATA TAAGTATTTA CATATGCAGT

PVY-P21 TGATTGTGAT GCCTCTCTCC GGACGATATA TAAGTATTTA CATATGCAGT

PVY-P2 TGATTGTGAT GC.TCTCTCC GGACGATATA TAAGTATTTA CATATGCAGT

51

*

100

PVY-Tu AAGTATTTTG GCTTTTCCTG TACTACTTTT ATTATAACTA ATAATCAGTT

PVY-P21 AAGTATTTTG GCTTTTCCTG TACTACTTTT ATTATAACTA ATAATCAGTT

PVY-P2 AAGTATTTTG GCTTTTCCTG TACTACTTTT ATCATAACTA ATAATCAGTT

\begin{tabular}{|c|c|c|c|c|c|}
\hline & .01 & & & & 150 \\
\hline & TGAATATTAT & TAATAGATAG & AGGTGGCAGG & GTGATTTCGT & CATTGTGG \\
\hline & TGAATATTAT & TAATAGATAG & AGGTGGCAGG & GTGATTTCGT & CATTGT \\
\hline 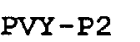 & TGAATATTAC & TAATAGATAG & AGGTGGCAGG & GTGATTTCGT & CATTG \\
\hline
\end{tabular}

151

200

PVY-Tu ACTCTATCTG TTAATTCTGC ATTATTAAGT TTTAGATAAA AGTGCCGGGT PVy-P21 ACTCTATCTG TTAATTCTGC ATtATTAAGT TTTAGATAAA AGTGCCGgGT PVY-P2 ACTCTATCTG TTAATTCTGC ATtATtAagt CTTAGATAAA AGTGCCGgGT

\begin{tabular}{|c|c|c|c|c|}
\hline & 201 & $\star$ & & \\
\hline PVY - Tu & TGTCGTTGTT & GTAGGTGAAC & CATCGATTAG & GTGA \\
\hline PVY - P21 & TGTCGTTGTT & GTAGGTGAAC & CATCGATTAG & GTGA \\
\hline \multirow[t]{2}{*}{$\mathrm{PVY}-\mathrm{P} 2$} & TGTCGTTGTT & GTGGGTGAAC & CATCGATTAG & GTGP \\
\hline & 251 & & & \\
\hline PVY -Tu & TAGCAGTGAC & TATGTCTGGA & TCTATCTACT & TGG \\
\hline PVY -P21 & TAGCAGTGAC & TATGTCTGGA & TCTATCTACT & TGE \\
\hline \multirow[t]{2}{*}{ PVY - P2 } & TAGCAGTGAC & TATGTCTGGA & TCTATCTACT & TGG \\
\hline & 301 & & & 333 \\
\hline PVY - Tu & TCATAACAGT & GACTGTAAAC & TTCAATCAGGA & $A G A$ \\
\hline$V Y-P 21$ & TCATAACAGT & GACTGTAAAC & TTCAATCAGGA & A $G$ \\
\hline$V Y-P 2$ & TCATAACAGT & GACTGTAAAC & TTCAATCAGGA & \\
\hline
\end{tabular}

totalement différente. La boucle IV située en position 296-302 est absente chez l'isolat PVY$P 2$. La boucle $V$ située en position 313 n'est présente que chez l'isolat PVY-P21.

Le rôle des structures "tige-boucle" de la région 3'NTR a été démontré dans les interactions spécifiques entre l'ARN viral et la protéine capsidiaire chez le phage R17 (Carey et al, 1983), dans la transactivation de la protéine «tat» chez le virus de l'immunodéficience humaine (HIV) (Feng et Holland, 1988), et dans l'induction des symptômes caractéristiques de l'infection virale chez le TVMV (Rodriguez-Cerezo et al, 1991). Chez les isolats tunisiens, la structure secondaire potentielle de la région 3 'NTR ainsi que la séquence de ses boucles semblent donc être impliquées dans l'expression des symptômes, ce qui pourrait expliquer l'apparition de nécroses sur les variétés de piment sensible
Beldi et Anaheim, uniquement suite à l'infection par l'isolat PVY-P21 (Fakhfakh et al, 1994).

\section{Analyse des séquences de la région capside}

Les séquences nucléotidiques des 3 isolats tunisiens ont été déterminées (fig 4). La comparaison de ces séquences montre que l'isolat PVYP21 se distingue des 2 autres isolats par 50 substitutions dont la position est indiquée par un astérisque. L'isolat PVY-Tu se distingue des autres isolats par 2 substitutions en position 630 et 705 . Le nucléotide en position 615 est différent chez les 3 isolats. Par ailleurs, parmi les sites de restriction polymorphes (17 enzymes de restriction impliquées), seule l'enzyme Bsrl possède un site de coupure au niveau de la séquence des isolats tunisiens sur piment (PVY-P2 et PVY- 


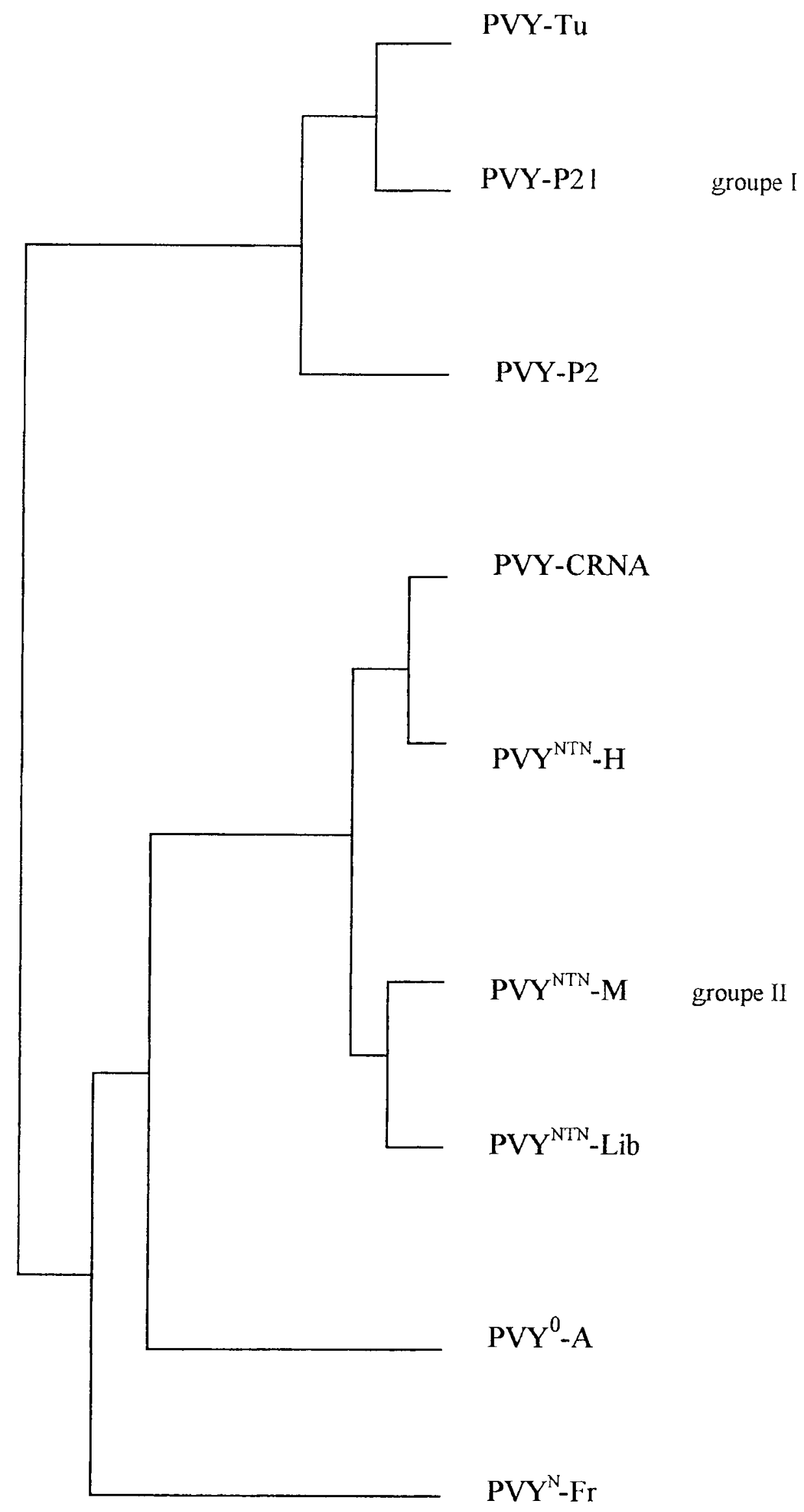

Fig 2. Dendrogramme établi à partir de l'alignement multiple des séquences en nucléotides de la région 3'NTR des différents isolats de PVY comparés. 


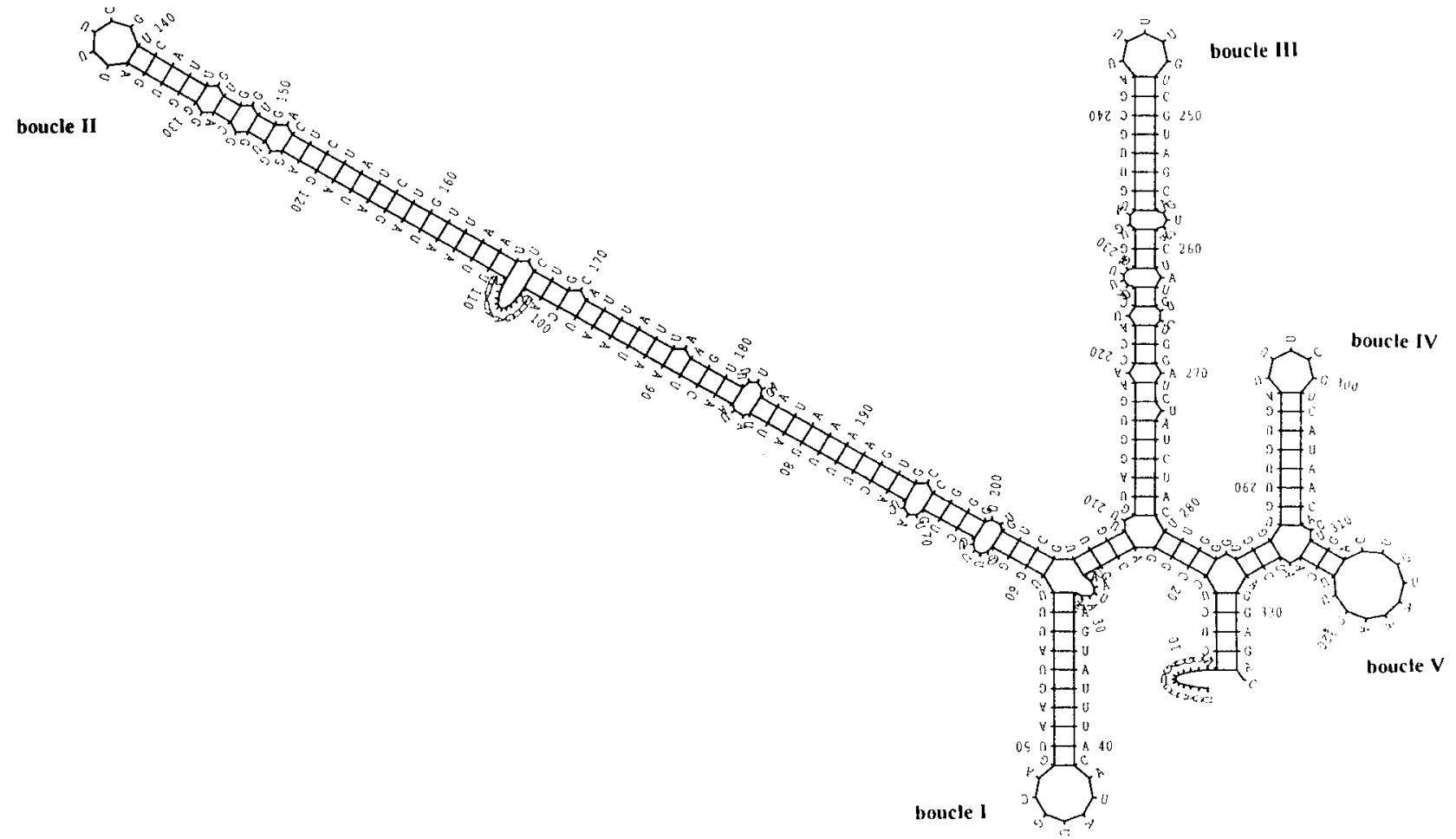

Fig 3. Structure secondaire potentielle de l'ARN viral de l'isolat PVY-P21 au niveau de la région 3 'NTR. Cette structure montrant 5 repliements en «tige-boucle» a été obtenue par le programme Fold du serveur GCG.

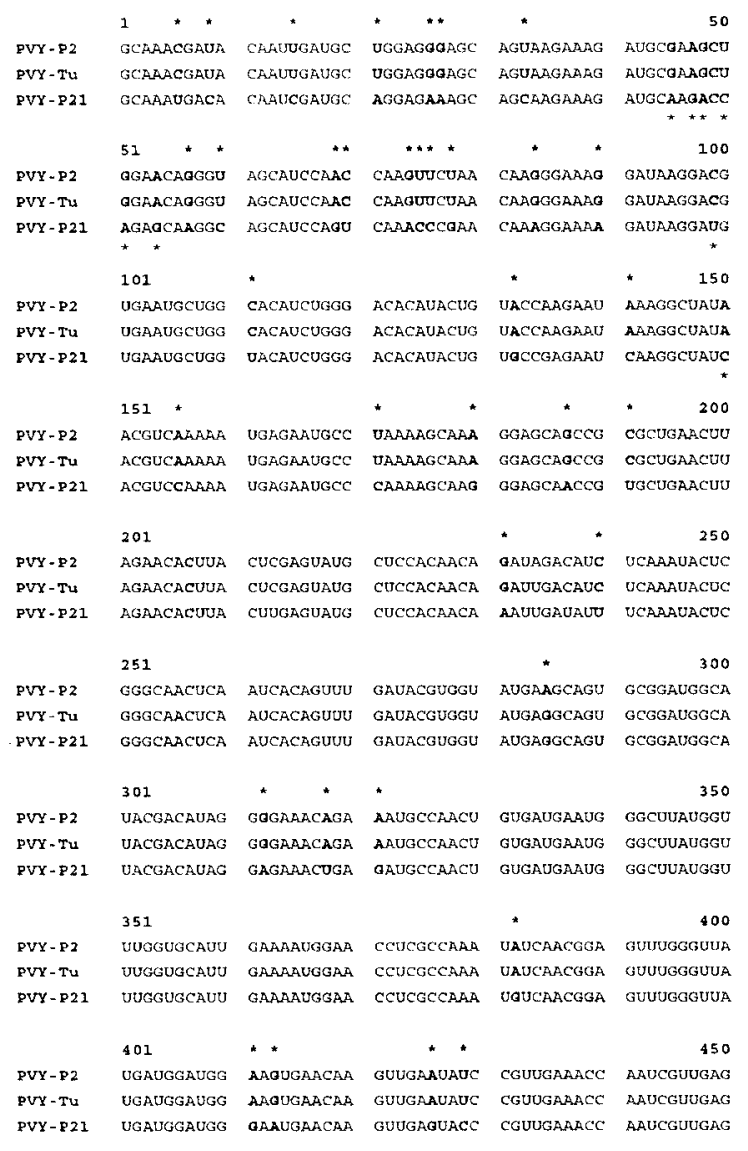

\begin{tabular}{|c|c|c|c|c|c|}
\hline & 451 & * & & & 500 \\
\hline PVY - P2 & AAUGCAAAAC & CGACCCUUAG & GCAALUCAUGG & GCACAUUUCU & CAGAUGUUGC \\
\hline PVY -Tu & AAUGCAAAAC & CGACCCUUAG & GCAAAUCAUG & GCACAUUUCU & CAGAUGUUGC \\
\hline \multirow[t]{2}{*}{ PVY - P21 } & AAUGCAAAAC & CAACCCUUAG & GCAAAUCAUUG & GCACAUUUCU & CAGAUGUUGC \\
\hline & 501 & & & & 550 \\
\hline PVY - P2 & AGAAGCGUAU & AUAGMAAUGC & GCAACAALAA & GGAACCAUAU & AUUGCCACGAU \\
\hline PVY - Tu & AGAAGCGUAU & AUAGAAAUGC & GCAACARAAA & GGAACCAUAU & AUGCCACGAU \\
\hline \multirow[t]{2}{*}{ pvY - P21 } & AGAAGCGUAU & AUAGAAAUGC & GCRACALAAA & GGAACCAUAU & AUGCCACGAU \\
\hline & 551 & & *** & & 600 \\
\hline $\mathrm{PVY}-\mathrm{P} 2$ & AUGGUUUAGU & UCGAAAUTUG & CGGGAUGGAA & GUUUAGCGCG & CUaUgccuUU \\
\hline PVY - Tu & AUGGUUUAGU & UCTAAAUUUUG & CGGGAUGAAA & GUUUAGCGCG & CUAUGSCCUUU \\
\hline \multirow[t]{2}{*}{$p v x-p 21$} & AUGGUUUAAU & $\begin{array}{l}\text { UCGAMAUCUG } \\
\star\end{array}$ & CGGGAUGUGG & GUUUAGCGCG & DUAUGCCUUU \\
\hline & 601 & * * & \multicolumn{2}{|c|}{ BorI } & 650 \\
\hline PVY - P2 & GACUUUUAUG & AAGUTACAUC & ACGAACACCA & GUGAGGGCUA & GAGAAGCGCA \\
\hline PVY - Tu & GRCuUvUaUG & AAGUAACAUC & ACGAACACACCC & GUGAGGGCUA & GAGAAGCGCA \\
\hline \multirow[t]{2}{*}{ PUY - D21 } & GACUUUTURUG & AQGUCACADC & ACGAACACCA & GUGAGGGCUA & GOGAAGCGCA \\
\hline & 651 & & & & 700 \\
\hline PVY - P 2 & UAUACAOAUG & AAGGCCGCAG & CAUTHAAAAUC & RGCCCAACCU & CGACUUUUCG \\
\hline$P V Y-T u$ & UAUACAOAUG & AAGGCCGCAG & CAUUAARAUC & AGCCCAAACCU & CGACUUUUCG \\
\hline \multirow[t]{2}{*}{ PVY - p21 } & $\begin{array}{l}\text { CAUUUCAARUG } \\
* * * *\end{array}$ & AAGGCCGCAG & CAUUUARAAUC & AGCCCARCCU & CGACUUUUCG \\
\hline & 701 & & & & 750 \\
\hline PVY - P2 & GGUUAGACGG & UGGCAUCAGU & ACACAAGAGG & AGAACACAGA & GAGGCACACC \\
\hline PVY - Tu & GGUUAGACGG & UGGCAJCAGU & ACACAAGAGG & AGAACACAGA & GAGGCACACC \\
\hline \multirow[t]{2}{*}{ PVY-P21 } & $\begin{array}{l}\text { GGUUGGACGG } \\
*\end{array}$ & UGGCAUCAGU & ACACAAGAGG & AGAACACAGA & GAGGCACACC \\
\hline & 751 & * & & & 800 \\
\hline PVY - P2 & ACCGAGGAUG & UVUCUCCAAG & UAUGCAUACU & CUACUUGGAG & UCAAGAACAU \\
\hline PVY - Tu & ACCGAGGAUG & UDUCUCCAAG & URUGCAUACU & CUACUUGGAG & UCAAGAACAU \\
\hline PVY - P21 & ACCGAGGAUG & UCUCUCCAAG & UAUGCAUACU & CUACUUGGAG & UCAAGAACAU \\
\hline & & & & & \\
\hline r-Tu & & & & & \\
\hline & & & & & \\
\hline
\end{tabular}

Fig 4. Alignement multiple des séquences en nucléotides de la région codant pour la protéine capside des isolats tunisiens. Les changements de nucléotides sont indiqués en gras et par un astérisque. 
P21) mais pas au niveau de la séquence de l'isolat tunisien d'origine pomme de terre (PVY-Tu).

Pour déterminer l'importance de ces changements nucléotidiques, les séquences en acides aminés de la protéine capside ont été déduites (fig 5). Ces séquences ont été comparées à celles de 6 autres isolats de PVY (tableau II).

L'étude comparative de la structure primaire de la protéine de la capside, prédite à partir de la séquence nucléotidique de tous les isolats, fait apparaître l'existence de domaines conservés et de domaines variables. Chez les 9 isolats comparés, la protéine capsidiaire est constituée de 268 résidus et le motif DAG impliqué dans la transmission par les pucerons est conservé. Toutes les séquences commencent par le motif consensus du PVY déterminé par plusieurs auteurs A/G,NDTIDAG... (Shukla et al, 1986 ; Robaglia et al, 1989 ; Rybicki et Shukla, 1992). D'autre part, les résultats obtenus montrent que pour les 3 isolats tunisiens, la séquence de la protéine capsidiale commence par le résidu (A), ce qui est en corrélation avec l'appartenance de l'isolat PVY-Tu au groupe $O$, comme il a été montré par Van der Vlugt et al (1993). Nos résultats montrent que la protéine capsidiale des isolats d'origine piment appartenant au pathotype 0 commence également par le résidu A. II est important également de remarquer la présence dans la séquence en acides aminés de l'isolat PVY-P21, de 13 changements par rapport à celles des 2 autres isolats tunisiens.

La comparaison de la structure primaire de la protéine capsidiaire avec celles d'autres isolats décrits dans la littérature permet de distinguer, au niveau du dendrogramme, 3 groupes selon le degré de similarité en acides aminés (fig 6). Le premier groupe comprend uniquement des isolats du type N. Le second rassemble les 2 isolats tunisiens PVY-Tu et PVY-P2, alors que dans le troisième, plus hétérogène, on remarque la présence d'isolats de différentes classification biologiques (type $\mathrm{N}$ et type $\mathrm{O}$ en même temps). Dans ce troisième groupe figure l'isolat PVY-P21, classé dans le pathotype 0 , qui n'est plus associé aux 2 autres isolats tunisiens. Un dendrogramme de même allure a été obtenu à partir de l'alignement global

\section{$\begin{array}{llcc}1 & * & * \star & * \star \\ \text { PVY-P2 } & \text { ANDTIDAGGS } & \text { SKKDAKLEQG } & \text { SIQPSSNKGK }\end{array}$ \\ PVY-TU ANDTIDAGGS SKKDAKLEQG SIQPSSNKGK \\ PVY-P21 ANDTIDAGES SKKDARPEQG SIQSNPNKGK \\ DKDVNAGTSG \\ DKDVNAGTSG \\ DKDVNAGTSG \\ THTVPRIKAI \\ THTVPRIKAI \\ TUTVPRIKAI}

51

PVY-P2 TSKMRMPKSK

PVY -TU TSKMRMPKSK

PVY-P21 TSKMRMPKSK

101

PVY - P2

PVY -Tu

PVY-P2I

$\begin{array}{ll} & 151 \\ \text { PVY-P2 } & \text { NAKPTLRQIM }\end{array}$

PVY-Tu NAKPTLRQIM

PVY-P21 NAKPTLRQIM

201

PVY-P2 DFYEVTSRTP

PVY-TU DFYEVTSRTP

PVY-P21 DFYEVTSRTP

251

PVY - P2

TEDVSPSMHT

PVY-Tu TEDVSPSMHT

PVY - P21

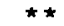

GAAALNLEHL

GAAALNLEHL

GATVLNLEHL

LEYAPQQIDI

LEYAPQQIDI

LEYAPQQIDI

$\star$
ENGTSPNING
ENGTSPNING
ENGTSPNVNG

VMNGLMVWCI

VMNGLMVWCI

VMNGLMVWCI

AHFSDVAEAY

AHFSDVAEAY

AHFSDVAEAY

IEMRNKKEPY

IEMRNKKEPY

IEMRNKKEPY

VRAREAHIQM

KAAALKSAQP

KAAALKSAQP

VRAREAHICM

KAAALKSAQP
100

SNTRATQSQF

SNTRATQSQF

SNTRATQSQF

DTWYEAVRMA

DTWYEAVRMA

DTWYEAVRMA

\section{0}

VWVMMDGSEQ VEYFLKPIVE

VWVMMDGSEQ VEYFLKPIVE

VWVMMDGNEQ VEYPLKPIVE

$\begin{array}{ccr}* & \star \star & 200 \\ \text { MPRYGLVRNL } & \text { RDGSLARYAF } \\ \text { MPRYGLVRNL } & \text { RDGSLARYAF } \\ \text { MPRYGLIRNL } & \text { RDVGLARYAF }\end{array}$

250

RLFGLDGGIS TQEENTERH RLFGLDGGIS TQEENTERHT RLFGLDGGIS TQEENTERHT
Fig 5. Alignement multiple des séquences en acides aminés (jusqu'au codon stop) de la région codant pour la protéine capside des isolats tunisiens. Les changements d'acides aminés sont indiqués en gras et par un astérisque. 


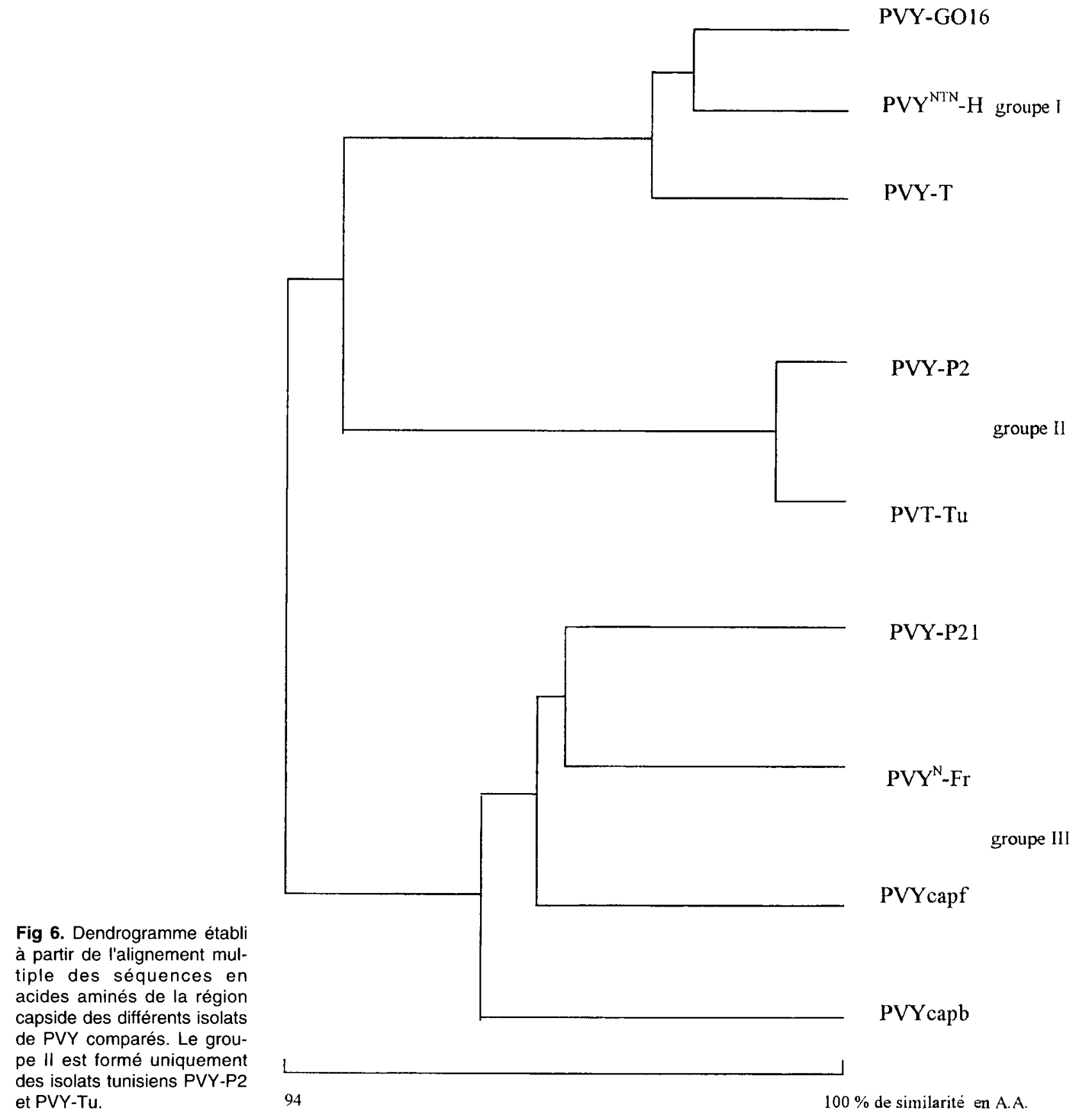

des séquences nucléotidiques (résultat non indiqué). L'ensemble de ces données suggère qu'un phénomène de recombinaison entre un isolat tunisien et un autre isolat de PVY serait à l'origine de l'isolat PVY-P21. Dans ce cadre, le fragment transféré devrait couvrir les nucléotides allant de la position 24 jusqu'à la position 582 de la région codant pour la protéine capside.

Le phénomène de recombinaison a été proposé par Goldbach (1992) pour expliquer l'origine de plusieurs variants de Potyviridae. De plus, en comparant la séquence de plusieurs isolats de PPV (plum pox virus), il a été montré que la région $\mathrm{C}$-terminale de la protéine $\mathrm{NIb}$ et $\mathrm{N}$-terminale de la protéine capside de l'isolat PPV-o6, proviendrait de la recombinaison entre 2 isolats sauvages de PPV (Cervera et al, 1993). Une situation analogue a été signalée chez des isolats de PVY d'origine pomme de terre. C'est le cas par exemple de la souche PVYn-Fr qui, sur la base de ses caractères biologiques et sérologiques est classée dans le groupe $\mathrm{N}$, alors que, sur la base de la séquence de la région capside, elle est classée dans le groupe $O$. II semble donc que les variations régissant l'évolution de ces isolats peuvent être soit «groupe-spécifique» $(\mathrm{N}, \mathrm{O})$, soit "souche-spécifique» quand elles se produisent au sein du même groupe (Van der Vlugt et al, 1993). 


\section{CONCLUSION}

Lors d'une étude précédente, 3 isolats tunisiens ont été classés sur la base d'une analyse biologique (Fakhfakh et al, 1994) et d'une analyse moléculaire des régions 5 'NTR et de la protéine P1 (Marie-Jeanne Tordo et al, 1995). Nous avons poursuivi cette étude en analysant les 2 régions $3^{\prime} \mathrm{NTR}$ et capside.

Les résultats obtenus pour la région 3'NTR sont en accord avec la classification biologique ainsi qu'avec les résultats obtenus lors de l'étude moléculaire au niveau des régions $5^{\prime} \mathrm{NTR}$ et la protéine P1. Ils confirment l'origine géographique commune des 2 isolats tunisiens d'origine piment et leur appartenance au pathotype 0 et de l'isolat tunisien d'origine pomme de terre appartenant au groupe $\mathrm{O}$.

Les différences observées au niveau des structures secondaires potentielles de la région 3'NTR du génome, montrant des structures tige-boucle, pourraient expliquer les différences symptomatologiques entre les 2 isolats inféodés au piment, en l'occurrence, les nécroses qui ne sont observées que lors de l'infection par l'isolat PVY-P21.

Les différences de séquence au niveau de la région capside de l'isolat PVY-P21 par rapport aux 2 autres isolats tunisiens suggèrent qu'un phénomène de recombinaison au niveau de cette région serait à l'origine de cet isolat. Ce dernier pourrait également provenir de l'un des 2 autres isolats suite à l'accumulation de mutations ponctuelles. Enfin, l'hypothèse que cet isolat soit introduit directement par les tubercules de pomme de terre n'est pas exclue.

L'isolat PVY-P2 aurait, quant à lui, divergé de l'isolat PVY-Tu suite à l'accumulation de certaines mutations principalement au niveau de la région 3'NTR.

Afin de confirmer ces hypothèses, il serait nécessaire d'explorer la totalité du génome de ces 3 isolats et d'analyser les effets pathologiques dus aux échanges de fragments entre les ADNc de ces 3 isolats. Par voie de conséquence, l'obtention d'un ADNc infectieux correspondant à la totalité du génome constitue une des priorités de nos recherches ultérieures.

\section{REMERCIEMENTS}

Nous remercions $C$ Cherif, $\mathrm{N}$ Hamza et $\mathrm{G}$ Khaldi de I'INRA de Tunisie pour leur aide à l'acquisition du matériel biologique. Nous remercions également la DGRST, le SERST (Tunisie), le CNRS, le ministère français des Affaires étrangères pour leur appui matériel dans la réalisation de ce travail et I'UNESCO pour nous avoir accordé une bourse de stage. Ce travail rentre dans la cadre du réseau formation-recherche franco-tunisien (projet Fac Sci Tunis/INRA).

\section{RÉFÉRENCES}

Atreya PL, Atreya CD, Pirone TP (1991) Amino acid substitutions in the coat protein result in loss of insect transmissibility of a plant virus. Proc Natl Acad Sci USA 88, 7887-7891

Bravo-Almonacid F, Mentaberry AN (1989) Nucleotide cDNA sequence coding for the $\mathrm{PVY}^{\circ}$ coat protein. Nucleic Acids Res 17, 4401

Brunt AA (1992) The general properties of potyviruses. Arch Virol (suppl 5), 3-16

Bryan GT, Gardner RG, Foster RLS (1992) Nucleotide sequence of the coat protein gene of a strain of clover yellow vein virus from New Zealand: conservation of a stem-loop structure in the $3^{\prime}$ region of potyviruses. Arch Virol 124, 133-146

Carey J, Cameron V, Haseth PL, Uhlenbeck OC (1983) Sequence-specific interaction of R17 coat protein with its ribonucleic acid binding site. Biochemistry 22, 2601-2610

Carrington JC, Dougherty WG (1987) Small nuclear inclusion protein encoded by a plant potyvirus genome is a protease. $J$ Virol $61,2540-2548$

Carrington JC, Cary SM, Parks TD, Dougherty WG (1989) A second proteinase encoded by a plant potyvirus genome. EMBO J 8, 365-370

Cervera MT, Riechmann JL, Martin MT, Garcia JA (1993) 3'-Terminal sequence of the plum pox virus PS and Ò6 isolates: evidence for RNA recombination within the potyvirus group. J Gen Virol 74, 329334

De Bokx JA, Huttinga $H$ (1981) Potato virus $Y$. CMI/AAB Descriptions of plant viruses, $n 242$

Devereux J, Haeberli P, Smithies O (1984) A comprehensive set of sequence analysis programs for the VAX. Nucleic Acids Res 12, 387-397

Dolja VV, Carrington JC (1992) Evolution of positivestrand RNA viruses. Sem Viro/ 3, 315-326

Dolja VV, Haldeman-Cahill R, Montgomery AE, Vandenbosch KA, Carrington JC (1995) Capsid protein determinants involved in cell-to-cell and long distance movement of tobacco etch potyvirus. Virology 206, 1007-1016

Dougherty WG, Hiebert E (1980) Translation of potyvirus RNA in a rabbit reticulocyte lysate: cell-free translation strategy and a genetic map of the potyviral genome. Virology 104, 183-194

Fakhfakh H, Makni M, Khadmaoui A, Cherif C, Elgaaied A, Marrakchi M (1994) Le virus Y de la pomme de terre dans les cultures de piment en 
Tunisie : identification et caractérisation des souches par voie biologique, préparation d'anticorps polyclonaux anti-PVY. Biologia 1, 52-69

Feng S, Holland EC (1988) HIV-1 tat trans-activation requires the loop sequence within tar. Nature 334, 165-167

Francki RIB (1983) Current problems in plant virus taxonomy. In : A Critical Appraisal of Viral Taxonomy (REF Matthews, ed), CRC Press, Boca Raton, 63-104

Francki RIB, Milne RG, Hatta T (1985) Atlas of Plant Virus, vol 2. CRC Press, Boca Raton, 183-217

Frenkel MJ, Ward CW, Shukla DD (1989) The use of $3^{\prime}$ non-coding nucleotide sequences in the taxonomy of potyviruses: application to watermelon mosaic virus 2 and soybean mosaic virus-N. $J$ Gen Virol 70, 2775-2783

Gébré Sélassié K, Marchoux G, Delécolle B, Pochard $E$ (1985) Variabilité naturelle des souches du virus $Y$ de la pomme de terre dans les cultures de piment du sud-est de la France. Caractérisation et classification en pathotypes. agronomie 5, 621-630

Goldbach R (1992) The recombinative nature of potyviruses: implications for setting up true phylogenetic taxonomy. Arch Virol (suppl 5), 299-304

Hari V (1981) The RNA of tobacco etch virus: further characterization and detection of protein linked to RNA. Virology 112, 391-399

Hidaka M, Yoshida $Y$, Masaki H, Namba S, Yamashita S, Tsuchizaki T, Uozumi T (1992) Cloning and sequencing of the $3^{\prime}$ half of potato virus $Y$ (O-strain) genome encoding the $5 \mathrm{~K}$ protein, protease, polymerase, and coat protein. Nucleic Acids Res 20, 3515

Hollings M, Brunt AA (1981) Potyviruses. In : Handbook of Plant Virus Infections and Comparative Diagnosis, 731-807

Lain S, Riechmann JL, Mendez E, Garcia JA (1988) Nucleotide sequence of the $3^{\prime}$ terminal region of plum pox potyvirus RNA. Virus Res 10, 325-342

Le Romancer M, Kerlan C, Nedellec M (1994) Biological characterization of various geographical isolates of potato virus $Y$ inducing superficial necrosis on potato tubers. Plant Pathol 43, 138-144

Marchuk D, Drumm M, Saulino A, Collins FS (1990) Construction of T-vectors, a rapid and general system for direct cloning of unmodified PCR products. Nucleic Acids Res 19, 1154

Marie-Jeanne Tordo V, Chachulska AM, Fakhfakh $\mathrm{H}$, Le Romancer M, Robaglia C, Astier-Manifacier S (1995) Sequence polymorphism in the 5'NTR and in the $P 1$ coding region of potato virus $Y$ genomic RNA. J Gen Virol 76, 939-949

Oshima K, Hataya T, Sano T, Inoue AK, Shikata E (1991) Comparaison of biological properties, serological characteristics and amino acid sequences of coat protein between potato virus $Y$ ordinary strain and necrotic strain. Ann Phytopathol Soc Jpn 57, 615-622
Riechmann JL, Lain S, Garcia JA (1992) Highlights and prospects of potyvirus molecular biology. $J$ Gen Virol 73, 1-16

Robaglia C, Durand-Tardif M, Tronchet $M$, Boudazin G, Astier-Manifacier S, Casse-Delbart F (1989) Nucleotide sequence of potato virus $Y$ (strain $N$ ). $J$ Gen Virol 70, 935-947

Rodriguez-Cerezo E, Gamble Klein P, Shaw JG (1991) A determinant of disease symptom severity is located in the 3-terminal non-coding region of the RNA of a plant virus. Proc Natl Acad Sci USA 88, 98639867

Rybicki EP, Shukla DD (1992) Coat protein phylogeny and systematics of potyviruses. In : Potyvirus taxonomy (OW Barnett, ed), Springer, Wien, 139-170 (Arch Virol suppl 5)

Sanger F, Nicklen S, Coulson AR (1977) DNA sequencing with chain-terminating inhibitors. Proc Natl Acad Sci USA 74, 5463-5469

Shukla DD, Inglis AS, McKern NM, Gouch KH (1986) Coat protein of potyvirus. 2. Amino acid sequence of the coat protein of potato virus Y. Virology 152, 118-125

Shukla DD, Ward CW (1988) Amino acid sequence homology of coat proteins as a basis for identification and classification of the potyvirus group. $J$ Gen Virol 69, 2703-2710

Shukla DD, Ward CW (1989) Identification and classification of potyviruses on the basis of coat protein sequence data and serology. Arch Virol 106, 171200

Steggles AW (1989) A rapid procedure for creating nested sets of deletions usings mini-prep plasmid DNA samples. Biotechniques 7, 241-242

Sudarsono, Woloshuk SL, Xiong Z, Hellmann GM, Wernsman EA, Weissinger AK, Lommel SA (1993) Nucleotide sequence of the capsid protein cistrons from six potato virus $Y$ (PVY) isolates infecting tobacco. Arch Virol 132, 161-170

Thole V, Dalmay T, Burgyan J, Balazs E (1993) Cloning and sequencing of potato virus $Y$ (Hungarian isolate) genomic RNA. Gene 123, 149-156

Turpen T (1989) Molecular cloning of a potato virus $Y$ : nucleotide sequence homology in non-coding regions of potyviruses. J Gen Virol 70, 1951-1960

Van der Vlugt RAA, Leunissen J, Golbach R (1993) Taxonomic relationships between distinct potato virus $Y$ isolates based on detailed comparisons of the viral coat proteins and $3^{\prime}$-nontranslated regions. Arch Virol 131, 361-375

Verchot JM, Koonin EV, Carrington JC (1991) The 35$\mathrm{kDa}$ protein from the $\mathrm{N}$ terminus of the potyviral polyprotein functions as a third virus-encoded proteinase. Virology $185,527-535$

Wefels E, Sommer H, Salamini F, Rohde W (1989) Cloning of the potato virus $Y$ genes encoding the capsid protein and the nuclear inclusion protein NIb. Arch Virol 107, 123-134 